\title{
Kinematics of Vega-like stars: Lifetimes and temporal evolution of circumstellar dust disks
}

\author{
P. Manoj and H. C. Bhatt \\ Indian Institute of Astrophysics, II Block, Koramangala, Bangalore 560034, India \\ e-mail: manoj@iiap.res.in \\ Received 10 May 2004 / Accepted 13 August 2004

\begin{abstract}
We have used the velocity dispersion as an age indicator to constrain the ages of a large sample of main sequence Vega-like stars in order to study the lifetimes and temporal evolution of the dust disks around them. We use Hipparcos measurements to compute stellar velocities in the sky plane. The velocity dispersion of Vega-like stars is found to be systematically smaller than that of the normal main sequence field stars for all spectral types, suggesting that the main sequence dusty systems, on average, are younger than normal field stars. The debris disks seem to survive longer around late type stars as compared to early type stars. Further, we find a strong correlation between fractional dust luminosity $\left(f_{\mathrm{d}} \equiv L_{\mathrm{dust}} / L_{\star}\right)$ and velocity dispersion of stars with dust disks. Fractional dust luminosity is found to drop off steadily with increasing stellar age from early pre-main sequence phase to late main sequence phase.
\end{abstract}

Key words. stars: circumstellar matter - stars: planetary system - stars: kinematics - stars: pre-main sequence - stars: evolution

\section{Introduction}

It has been well established that the majority of pre-main sequence stars are surrounded by circumstellar disks which are analogous in their properties to the protosolar nebula before the onset of planet formation (e.g., Beckwith 1999; Mundy et al. 2000; Wilner \& Lay 2000; Beckwith \& Sargent 1996). Young circumstellar disks lose most of this material due to planet formation and other disk dispersal processes by the time the central stars harbouring these disks reach the main sequence. In the standard model of planet formation, the dust grains with sizes typical of interstellar dust settle down to the disk midplane and stick together to grow into rocky planetesimals (e.g. Weidenschilling \& Cuzzi 1993). The disk is depleted of smaller grains and this lowers the opacity of the reprocessing disk. Planetesimals grow further to earth-like planetary bodies by coalescence and eventually accrete gas in the outer disk to form giant planets (e.g. Pollack et al. 1996). When the disk has become sufficiently gas-free, so that it is dominated by grain dynamics, the planetary mass objects can gravitationally perturb kilometer-sized planetesimals sending them into highly eccentric orbits. Collisions between these planetesimals then replenish the disk with "second generation dust" which is observed around many main sequence stars (e.g. Lagrange et al. 2000). These main sequence stars with debris disks, known as "Vegalike" stars, were first discovered by IRAS in 1983 (Aumann et al. 1984). At least $15 \%$ of main sequence stars are surrounded by such disks (Lagrange et al. 2000).

Planet formation is well underway in main sequence dusty systems and the disks that we observe are the debris product of the planet formation process (Lagrange et al. 2000). These disks are thought to be "sign posts" of planet formation (Kenyon \& Bromley 2002). What are the lifetimes of these disks? Do disk lifetimes depend on the nature of the central star? How do these observed disks evolve with time? These questions are central to our understanding of planet formation and disk evolution. A study of the lifetimes and the temporal evolution of the dust disks should provide insight into the formation of planetary systems and disk dispersal timescales and mechanisms.

There have been a number of studies on the evolution of circumstellar disks around main sequence stars. Zuckerman \& Becklin (1993) have found that the mass of dust in the disks declines as rapidly as (time) $)^{-2}$ during the initial $3 \times 10^{8} \mathrm{yr}$. Similar results have been reported by Holland et al. (1998) from their SCUBA observations. There is general agreement now on the fact that the amount of dust retained in the disks decreases with increasing stellar age (Habing et al. 1999; Lagrange et al. 2000). However, the exact nature of this decline is not clear. Most of these studies are based on the ages of a few prototype Vega-like stars. When more stars are employed, reliable estimation of their ages poses a serious problem.

It is difficult to determine the ages of main sequence stars with reasonable accuracy. There have been a few attempts to estimate ages of field Vega-like stars (e.g. Lachaume et al. 1999; Song 2000; Silverstone 2000), but the ages determined using different techniques are not always mutually consistent (Zuckerman 2001). Recently, Spangler et al. (2001) have carried out a survey of circumstellar disks around pre-main 
sequence and young main sequence stars that are members of young open clusters of known ages using ISOPHOT. They found that the fractional dust luminosity $f_{\mathrm{d}}$ drops off with stel-

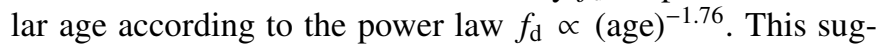
gestion of a global power law has been contested by Decin et al. (2003) who find a spread in fractional dust luminosity at any age from the revised age estimates of their sample stars observed by ISOPHOT. Dominik \& Decin (2003), based on a physical model that they developed for the dust production in Vega-like disks, have argued that a collisional cascade with constant collision velocities leads to a power law decrease of the amount of dust seen in the debris disk with a power law index of -1 . They add that a collisional cascade with continuous stirring can produce slopes steeper than -1 .

In this paper, we study the temporal evolution of dust disks around main sequence stars. We consider the kinematics of a large sample of Vega-like stars and use the velocity dispersion as an age indicator. It has long been known that there is a strong correlation between the random velocities and ages of stars in the Galactic disk. Velocity dispersion of stars in the solar neighborhood has been found to increase with age (Wielen 1977; Jahreiß \& Wielen 1983). Observationally, velocity dispersion $\sigma$ is found to grow with age at least as fast as $t^{0.3}$ and more likely $t^{0.5}$ (Wielen 1977; Binney \& Tremaine 1987). The dynamical origin of this effect is attributed to the encounters between the disk stars and the massive gas-clouds (Spitzer \& Schwarzschild 1951, 1953) and to transient spiral waves heating up the Galactic disk (Barbanis \& Woltjer 1967). Using accurate Hipparcos parallaxes and proper motions, Binney et al. (2000) and Dehnen \& Binney (1998) have shown that for a coeval group of stars, the rms dispersion in transverse velocity $S$ (in the plane of the sky), which is connected to the principal velocity dispersion by the relation $S^{2}=2 / 3\left[\sigma_{R}^{2}+\sigma_{\phi}^{2}+\sigma_{z}^{2}\right]$, increases with time from $8 \mathrm{~km} \mathrm{~s}^{-1}$ at birth as $t^{1 / 3}$. We follow this formalism and use the dispersion in transverse velocity to constrain the ages of Vega-like stars in order to study the lifetimes and temporal evolution of the dust disks.

\section{Data}

A number of recent studies give lists of Vega-like stars and candidate Vega-like stars, selected on the basis of their infrared excesses in the IRAS wave bands. Song (2000) lists 361 objects taken from different surveys and searches published in the literature. From a search of the IRAS FSC catalog, Silverstone (2000) produced a list of 191 Vega-like stars. A number of additional Vega-like objects have been discussed in Coulson et al. (1998) and Malfait et al. (1998). We first compiled a total of 486 distinct Vega-like stars taken from these lists that had many objects in common. This large sample could have some stars that are erroneously classified as Vega-like or have uncertain associations with the IRAS sources due to the large IRAS beam size. In our study, we consider only those stars for which the positional offset between the optical star and the IRAS association is $\leq 30^{\prime \prime}$. Further, we examined the Digital Sky Survey images of the region near each Vega-like candidate to make sure that the far-infrared emission is indeed from the stellar source and not from any extended background source, for example a galaxy, near the star in the plane of the sky (e.g., Zuckerman $\&$ Song 2004). We have eliminated those stars from our sample where an optical galaxy or an IRAS extended source was found inside or very near $\left(\leq 1^{\prime}\right)$ to the IRAS error ellipse for the point source. Alternative associations have been found for some of the proposed Vega-like stars in the literature (Sylvester \& Mannings 2000; Lisse et al. 2002). We have excluded such stars from our sample. We also exclude known pre-main sequence stars (e.g., Herbig Ae/Be stars; Thé 1994) and other emission-line objects from our sample. Further, we consider only stars in the spectral range between B9 and K5 - infrared excess from early B type star could be due to free-free emission (Zuckerman 2001) and K-giants are known to exhibit Vega-like excesses (e.g., Plets et al. 1997; Jura 1999). Finally, our sample contains 221 Vega-like stars for which both Hipparcos and IRAS (PSC/FSC) measurements are available.

\section{Analysis}

\subsection{Dustiness of Vega-like stars}

A good measure of the "dustiness" of the disks around Vegalike stars is the fractional dust luminosity $f_{\mathrm{d}} \equiv L_{\text {dust }} / L_{\star}$, which represents the optical depth offered by an orbiting dust disk to ultraviolet and visual radiation (Zuckerman 2001). We compute $f_{\mathrm{d}}$ from IRAS (PSC/FSC) fluxes for the Vega-like stars in our sample using the relation

$$
\begin{aligned}
f_{\mathrm{d}} & =L_{\text {dust }} / L_{\star} \\
& =\frac{10^{-4} \times\left[6.45 e_{12}+2.35 e_{25}+1.43 e_{60}+0.55 e_{100}\right]}{10^{\left[0.4\left(4.75-m_{\mathrm{V}}-B C\right)\right]}}
\end{aligned}
$$

(Emerson 1988). In the above equation $e_{12}, e_{25}, e_{60}, e_{100}$ are the excess flux densities in Jy over the photospheric values in the IRAS wave bands at 12, 25, 60 and $100 \mu \mathrm{m}$ respectively, $m_{\mathrm{V}}$ is the visual magnitude of the star corrected for extinction (which is generally very small, typically $\leq 0.1 \mathrm{mag}$ for stars in our sample) and BC is the bolometric correction.

The excess flux density in each IRAS band was estimated as follows. The photospheric $12 \mu \mathrm{m}$ magnitude was derived from the extinction-corrected $V$ magnitude and $(B-V)$ color of the star as discussed in Oudmaijer et al. (1992). The photospheric magnitudes at the other bands were calculated using the relations given in Oudmaijer et al. (1992). Photospheric magnitudes were then converted into flux densities in the IRAS bands by using the magnitude zero points listed in the IRAS catalog, and then color corrected to a photospheric SED (e.g., Silverstone 2000). The photospheric estimates in the IRAS catalog color convention were subtracted from the corresponding non-upper limit IRAS PSC/FSC flux densities to obtain the excess flux densities $e_{\lambda}$ in each of the IRAS bands.

In order to account for the possible inexact approximation of the photosphere, the excesses computed are considered, as in Silverstone (2000), to be significant only if it exceeds $20 \%$ of the photospheric flux value in each of the bands. Stars with significant excess in any one of the bands are taken as "true" Vega-like and their $f_{\mathrm{d}}$ is computed as described above. Fractional dust luminosities thus computed for 181 "true" 
Vega-like systems agree well with earlier estimates in the literature (Backman \& Gillett 1987; Song 2000; Silverstone 2000), generally to within $10 \%$. We find a number of stars with excesses of less than $20 \%$ of the photospheric fluxes in all the four IRAS bands. The excesses, if any, that these stars show are at a very low level. However, these stars have been classified as Vega-like stars in earlier studies in which excesses were inferred by different methods. We assign an upper limit value of $10^{-6}$ for $f_{\mathrm{d}}$ of these stars as the values of $f_{\mathrm{d}}$ computed for them are $\lesssim 10^{-6}$.

\subsection{Kinematics - transverse velocities of Vega-like stars}

For all the stars in our sample we have proper motions and parallaxes from the Hipparcos catalog (ESA 1997). The transverse velocity perpendicular to the line of sight relative to the solar system barycenter is then computed using the relation $V_{T}=\frac{A_{\mathrm{v}} \mu}{\pi}$ where $A_{\mathrm{v}}=4.740470 \mathrm{~km} \mathrm{yr} \mathrm{s}^{-1}, \pi$ is the parallax in milliarcseconds and $\mu=\left(\mu_{\delta}^{2}+\left(\mu_{\alpha} \cos \delta\right)^{2}\right)^{1 / 2}$ with $\mu_{\delta}$ and $\mu_{\alpha} \cos \delta$ being the proper motions along declination and right ascension in milliarcseconds. Errors in transverse velocities are estimated from the probable errors in parallaxes and proper motions given in the Hipparcos catalog. Transverse velocities of stars thus obtained will have solar motion reflected in them. We have corrected the velocities for solar motion using the values of $U=10.0 \pm 0.4 \mathrm{~km} \mathrm{~s}^{-1}, V=5.2 \pm 0.6 \mathrm{~km} \mathrm{~s}^{-1}$, $W=7.2 \pm 0.4 \mathrm{~km} \mathrm{~s}^{-1}$ (Binney \& Merrifield 1998) for the standard solar motion. To minimize the effect of Galactic differential rotation we consider only stars within $250 \mathrm{pc}$ from the Sun. Further, we include only those stars in our analysis that have a fractional error in transverse velocity less than 0.5. We have, then, 158 Vega-like stars with transverse velocities and fractional dust luminosities computed for the final analysis.

\section{Results}

\subsection{Velocity dispersion and disk lifetimes}

As discussed in Sect. 1, the velocity dispersion of a group of stars is a measure of the average age of the group. Here we use transverse velocity dispersion as an age indicator to constrain the ages of the stars with dusty disks in order to study the disk lifetimes and evolution. The transverse velocity dispersion of the 158 Vega-like stars in our final sample is found to be $21.4 \pm 1.2 \mathrm{~km} \mathrm{~s}^{-1}$. The transverse velocity dispersion, again computed using Hipparcos measurements, of about 14000 field stars with spectral types between B9 and K5 which are within $250 \mathrm{pc}$ from the Sun and whose fractional errors in transverse velocities are less than 0.5 , is computed to be $37.3 \pm 0.2 \mathrm{~km} \mathrm{~s}^{-1}$. The smaller velocity dispersion for Vega-like stars compared to field stars indicates, at the very outset, that the main sequence dusty systems are younger than the field stars and that the debris disk lifetimes are shorter than the main sequence lifetimes of the stars. In the following, we analyze the spectral type dependence of the velocity dispersion of Vega-like stars.

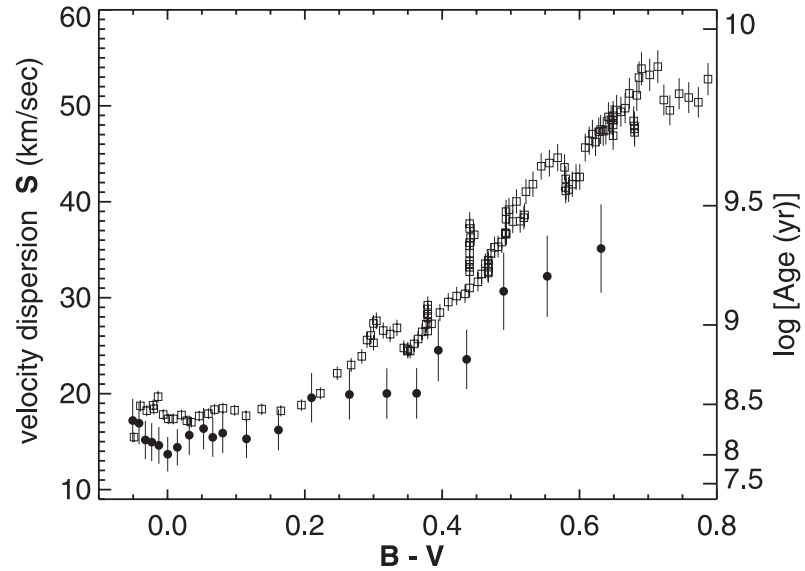

Fig. 1. Transverse velocity dispersion $S$ plotted against the dereddened color $(B-V)$ for Vega-like stars and normal field stars. Filled circles represent Vega-like stars and open squares the normal field stars. The vertical error bars are errors in velocity dispersion. The ages derived from the velocity dispersion using the formalism of Binney et al. (2000) is also shown on the y-axis on the right. Note that the age axis is not linear.

In Fig. 1 we plot the velocity dispersion of Vega-like stars against their dereddened $(B-V)$ color. The values of transverse velocity dispersion $\mathrm{S}$ shown in Fig. 1 are for a sliding window of 30 stars plotted against the mean $(B-V)$ for each bin. A fresh point is plotted every time six stars have left the window. We have also plotted the velocity dispersion of the field stars, computed from their Hipparcos proper motions and parallaxes in the same way as that for Vega-like stars, for comparison. The values of S plotted for field stars are for bins of 500 stars with a new point plotted when 100 stars have left the window. The error bars plotted in $\mathrm{S}$ are the standard deviation of dispersion in each bin which is given by $\Delta S=S / \sqrt{(2 n-2)}$ where $n$ is the number of stars in the bin.

It can be seen from Fig. 1 that the Vega-like stars, on average, show a lower velocity dispersion than the field stars at any given $(B-V)$. While this trend is clearly evident on visual inspection for stars with $B-V \geq 0.3$ (spectral type $\mathrm{F} 0$ or later), it is not as striking for stars of early spectral type, though in general, their velocity dispersions are smaller than the field stars. A two-sided two dimensional Kolomogorov-Smirnov test shows that the velocity dispersion $-(B-V)$ relation for Vegalike stars and field stars to be different with probability $99.99 \%$. Thus, Vega-like stars have a lower velocity dispersion than that of field stars for any given spectral type. Since a lower velocity dispersion indicates younger ages, it follows that the main sequence stars with debris disks are statistically younger than the field stars of similar spectral type. It can also be seen from Fig. 1 that late type Vega-like stars have a larger velocity dispersion than early type Vega-like stars, suggesting that statistically they are older.

In order to quantify the disk lifetimes of Vega-like stars, we relate the velocity dispersion to the stellar age following the formalism of Binney et al. (2000) where the velocity dispersion for a coeval group of stars as a function of age is given by $S=v_{10}\left[\left(\tau+\tau_{1}\right) /\left(10 \mathrm{Gyr}+\tau_{1}\right)\right]^{\beta}$. In this equation, $\tau_{1}$ determines the random velocity of stars at birth, and $v_{10}$ and $\beta$ 
characterize the efficiency of stellar acceleration. Using values of $\beta=1 / 3, v_{10}=58 \mathrm{~km} \mathrm{~s}^{-1}$ and $\tau_{1}=0.03 \mathrm{Gyr}$ (Binney et al. 2000) we translate velocity dispersion into age. The ages thus derived from the velocity dispersion are plotted in Fig. 1 on the $y$-axis on the right. The figure shows Vega-like stars to be systematically younger than the field stars for all values of $(B-V)$.

Vega-like stars being a younger population than the field stars indicates that the lifetimes of debris disks are shorter than the main sequence lifetimes of the stars which harbour them. The debris disk may not survive for the entire lifetime of the central star. However, it is possible that this is a selection effect, as IRAS will not be able to detect low luminosity disks below its sensitivity limit. The luminosity of debris disks gradually goes down as the central stars age and eventually falls below the limit of detection. This is expected physically as the larger bodies which replenish the disk are eroded continuously and are finite in supply. We also find such a fall in fractional dust luminosity of debris disks with age as demonstrated in Fig. 2 (see Sect. 4.2). Dust debris of low optical depth $f_{\mathrm{d}} \lesssim 10^{-7}$ like that around the Sun may be present over the entire lifetimes of the main sequence stars.

The ages of Vega-like stars are found to range from $10^{8} \mathrm{yr}$ to 1-2 Gyr with Vega-like stars of later spectral types being older on average than stars of early spectral types. Ages of early type Vega-like stars span from $10^{8}$ yr to $4-5 \times 10^{8}$ yr while late type Vega-like stars can be as old as 1-2 Gyr. That main sequence dusty systems of earlier spectral type are systematically younger than the late type systems strongly suggests shorter lifetimes for debris disks around early type stars. This would mean that the temporal evolution of main sequence disks is a function of the spectral type of the central star, with debris disks surviving longer around late type stars, a conclusion also reached by Song (2001). This is not surprising as the timescales for grain removal processes like radiation pressure "blowout" and Poynting-Robertson drag which dominate Vega-like disks are inversely proportional to the stellar luminosity, and thus are shorter for early type stars.

\subsection{Temporal evolution of dust disks}

Next, we study the evolution of the "dustiness" of circumstellar disks with age. We look for a correlation between fractional dust luminosity $f_{\mathrm{d}}$ and transverse velocity dispersion $S$, for stars of similar $f_{\mathrm{d}}$. For this we grouped the stars into bins of a given range in dustiness $\left(f_{\mathrm{d}}\right)$. We then computed the dispersion in transverse velocities of stars in each of these bins. The mean value of $f_{\mathrm{d}}$ in each bin is then plotted against the velocity dispersion of stars in that bin as shown in Fig. 2. Error bars for $f_{\mathrm{d}}$ represent the standard deviation from the mean in each bin. Error bars in $\mathrm{S}$ are computed in the same way as that in Fig. 1. The point with a downward arrow represents all stars for which the computed $f_{\mathrm{d}} \leq 10^{-6}$ (see Sect. 3.1). In Fig. 2, we also include Herbig Ae/Be stars which are premain sequence stars of intermediate mass and are thought to be the progenitors of Vega-like stars. We computed $V_{T}$ and $f_{\mathrm{d}}$ for 44 Herbig Ae/Be stars taken from Thé et al. (1994), van den Ancker et al. (1998) and Malfait et al. (1998), and for

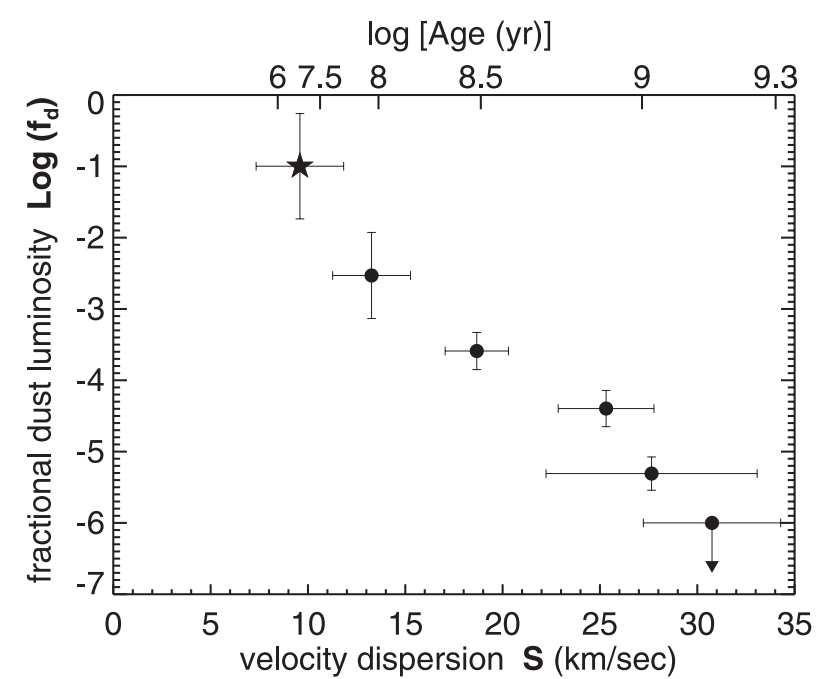

Fig. 2. Fractional dust luminosity $f_{\mathrm{d}}$ plotted against transverse velocity dispersion $S$ for stars with circumstellar dust disks. Filled circles represent Vega-like stars. Herbig Ae/Be stars are also plotted, represented by the solid star symbol. The point with a downward arrow represents stars with $f_{\mathrm{d}} \leq 10^{-6}$ (see text). Horizontal error bars represent errors in velocity dispersion. The ages appropriate for the velocity dispersion are also shown on the $x$-axis on the top. Note that the age axis is not linear.

which Hipparcos and IRAS measurements are available. As for Vega-like stars, we restrict ourselves to stars within $250 \mathrm{pc}$ and with fractional errors in transverse velocities less than 0.5 . We have 22 such Herbig Ae/Be stars. Their average $f_{\mathrm{d}}$ is plotted against the velocity dispersion $S$ and is represented by the filled star symbol in Fig. 2.

It is clear from Fig. 2 that there is a systematic decrease in the dustiness $\left(f_{\mathrm{d}}\right)$ of the disks with increasing velocity dispersion $S$ of stars. Herbig Ae/Be stars have the velocity dispersion $S$ smaller than that of Vega-like stars, indicating younger ages. As discussed earlier, the velocity dispersion $S$ of stars, in general, is found to increase with stellar age as $S \propto t^{1 / 3}$ (Binney et al. 2000). The correlation between fractional dust luminosity of stars with disks and their velocity dispersion seen in Fig. 2 clearly implies a steady decrease in the optical thickness of the disks with stellar age. This is consistent with the earlier findings that the amount of dust in the disks appears to decrease generally with system age.

The stellar ages obtained from translating velocity dispersion into age using the formalism of Binney et al. (2000) are also shown in Fig. 2 on the $x$-axis on the top. The steady drop of fractional dust luminosity with increasing stellar age is evident from the figure. There is an overall decrease in the "dustiness" of the circumstellar disks from the early pre-main sequence phase (a few Myr) to well up to the late main sequence phase ( $\sim 1-2$ Gyr). It can also be seen from Fig. 2 that the Herbig Ae/Be stars have larger values of $f_{\mathrm{d}}$ and younger ages than Vega-like stars. This is consistent with them being the progenitors of Vega-like stars.

We note here that the correlation that we find between $f_{\mathrm{d}}$ and velocity dispersion is not because of the spectral type dependence of velocity dispersion that we discussed earlier. Such 


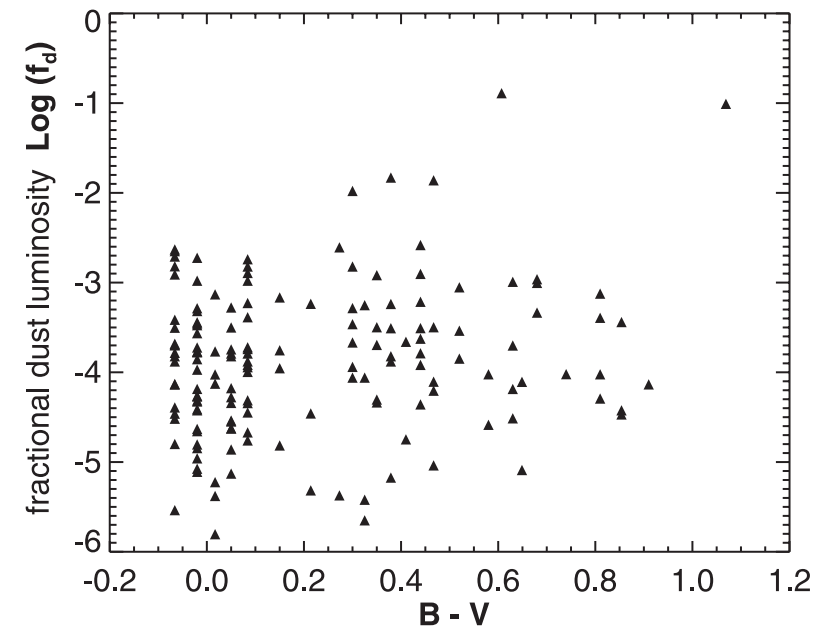

Fig. 3. Fractional dust luminosity $f_{\mathrm{d}}$ plotted against dereddened color $(B-V)$ for Vega-like stars.

a manifestation is expected if $f_{\mathrm{d}}$ has a spectral type dependence where the late type stars preferentially have lower values of $f_{\mathrm{d}}$. However, for our sample stars, we find that there is no such trend of $f_{\mathrm{d}}$ with spectral type. In Fig. 3 we present a plot of $f_{\mathrm{d}}$ against $(B-V)$ where it can be seen that there are as many or more early type stars with lower values of $f_{\mathrm{d}}$ as there are late type stars.

It should also be noted that the ages that we derive from the velocity dispersion are statistical in nature. They are the average ages appropriate for the velocity dispersion shown by the group of stars and are derived from the relation between velocity dispersion and age as given by Binney et al. (2000). The ages obtained this way can have relatively large errors for small ages because for small ages the velocity dispersion $S$ is a steeply rising function of age for the relation $S \propto t^{1 / 3}$.

\section{Discussion}

Of the 158 stars in our final sample for which the analysis was carried out, $107(68 \%)$ are within $100 \mathrm{pc}$ and $51(32 \%)$ have $d>100$ pc. Kalas et al. (2002) have cautioned that the far-infrared emission from some of the Vega-like stars beyond $100 \mathrm{pc}$ could be thermal emission from interstellar reflection nebulosities, similar to that seen in the Pleiades, due to the chance encounters of stars with relatively dense interstellar clouds. The dust density $\rho$ of the reflection nebulosities required to account for the observed fluxes is in the range of $10^{-24}-10^{-23} \mathrm{~g} \mathrm{~cm}^{-3}$ (Kalas et al. 2002) which corresponds to a gas number density $n$ of $10^{2}-10^{3} \mathrm{~cm}^{-3}$. Such high densities are generally associated with molecular clouds and not with the normal interstellar HI clouds. In fact the reflection nebulosity which causes the so called "Pleiades phenomenon" is a fragment of the Taurus-Auriga molecular cloud that has been encountered by the Pleiades in that cluster's southward motion (Herbig \& Simon 2001). Such encounters of stars with dense molecular cloud clumps in the solar neighbourhood must be rare. Of the initial sample of 79 Vega-like candidates of Kalas et al. (2002), 72 have Hipparcos-detected distances, and of these $43(60 \%)$ have $d>100 \mathrm{pc}$. Among the 60 stars that they have observed from this sample, only six are found to have reflection nebulosities surrounding them. We have excluded these stars from our sample. Therefore amongst the stars in our sample that have not yet been investigated for the "Pleiades phenomenon", one would expect only a few additional objects with reflection nebulosities. Because our analysis is statistical in nature, the average trends that we derive are not expected to be affected, even if a few stars with $d>100 \mathrm{pc}$ are found to be surrounded by reflection nebulosities instead of orbiting dust grains. Restricting our entire analysis to stars within $100 \mathrm{pc}$ gives the same trends and does not affect the results, except for poorer number statistics.

In Fig. 2 we have plotted both Herbig Ae/Be stars and Vega-like stars together and have derived an overall decline in fractional dust luminosity with stellar age. We point out here that the pre-main sequence disks like those around Herbig $\mathrm{Ae} / \mathrm{Be}$ stars and the main sequence disks are physically different. Pre-main sequence disks are relatively optically thick $\left(f_{\mathrm{d}} \sim 0.1\right)$ and gas-rich and are formed from the primordial cloud core from which the star itself is born. The infrared excess that the IRAS detected in these disks is due to the reradiation from the first generation dust grains. On the other hand, the infrared excess shown by Vega-like disks is due to the debris dust produced in the collisions between larger bodies. The debris disks are gas-poor and the disk evolution is dominated by dust dynamics. Moreover, the decline in the fractional dust luminosity in the pre-main sequence disks is primarily due to the grain growth by which smaller sub-micron sized grains get depleted in the disks thereby reducing the effective surface area of dust absorption/emission. In main sequence debris disks, where the grains causing infrared excess are continuously being replenished, the fall in $f_{\mathrm{d}}$ is due to the decline in the collisional regeneration rate.

Nevertheless, it is expected that the pre-main sequence disks gradually evolve into Vega-like disks, although it is not yet clear when exactly the secondary dust generation begins in these disks. Recent studies have suggested that in general, the lifetimes of primordial disks are only a few Myr (Haisch et al. 2001; Lada 1999). Larger bodies like kilometer-sized planetesimals and comets, which replenish the main sequence debris disks, can also be formed within a few Myr (e.g. Beckwith et al. 2000). Thus the transition of optically thick disks into optically thin disks is expected to take place on similar timescales. The resolution of the stellar ages derived from velocity dispersion is poor and is inadequate to address the issue of the timescale of the transition from primordial pre-main sequence disks to Vega-like disks. However, a general decline in the "dustiness" of the disks with stellar age from the pre-main sequence phase to the late main sequence phase is clearly seen.

The results that we obtain from our analysis are consistent with the earlier studies on the disk evolution around main sequence stars. The overall decline in the dust content of the debris disks has been reported by Zuckerman \& Becklin (1993), Holland et al. (1998) and Spangler et al. (2001). The conclusions of Decin et al. (2003) that there are few young stars with small excesses and that a Vega-like excess is more common in young stars than in old stars are consistent with our result of Vega-like stars being younger than the field stars for all spectral 
types. Decin et al. (2003) also argue that at most ages, there is a spread in fractional dust luminosity of Vega-like stars. While this may be true, our results strongly suggest that there is also a general decline in the $f_{\mathrm{d}}$ of the disks with stellar age. However, we are not able to fit a single power law to the fall in $f_{\mathrm{d}}$ with the average stellar ages that we derive from the velocity dispersion of the stars.

\section{Conclusions}

In this paper we have used velocity dispersion as an age indicator to constrain the ages of a large sample of Vega-like stars. From the statistical ages derived from the velocity dispersion, we have studied the disk lifetimes and the temporal evolution of the dust disks around main sequence stars. The conclusions of this study are summarized below.

- Velocity dispersion of Vega-like stars is found to be smaller than that of main sequence field stars for all spectral types. Main sequence stars with debris disks, on average, are younger than normal field stars of similar spectral type.

- The ages of Vega-like stars derived from the velocity dispersion range from $10^{8} \mathrm{yr}$ to $1-2 \mathrm{Gyr}$.

- Vega-like stars of later spectral types are statistically older than Vega-like stars of earlier spectral type. Debris disks seem to survive longer around late type stars as compared to early type stars.

- There is a strong correlation between fractional dust luminosity and velocity dispersion of Vega-like stars. Average fractional dust luminosity $f_{\mathrm{d}}$ of stars with disks decreases monotonically with increasing velocity dispersion. There is a general decline of $f_{\mathrm{d}}$ with stellar age from the early premain sequence phase to the late main sequence phase.

- The observed high $f_{\mathrm{d}}$, lower velocity dispersion $S$ and the implied younger ages for Herbig Ae/Be stars are consistent with them being the progenitors of Vega-like stars.

\section{References}

Aumann, H. H., Beichman, C. A., Gillett, F. C., et al. 1984, ApJ, 278, L23

Backman, D., \& Gillett, F. C. 1987, in Cool Stars, Stellar Systems and the Sun, LNP, 291, 340

Barbanis, B., \& Woltjer, L. 1967, ApJ, 150, 461

Beckwith, S. V. W. 1999, in The Origin of Stars and Planetary Systems, NATO ASIC Proc., 540, 579

Beckwith, S. V. W., Henning, T., \& Nakagawa, Y. 2000, Protostars and Planets IV, 533

Beckwith, S. V. W., \& Sargent, A. I. 1996, Nature, 383, 139

Binney, J., Dehnen, W., \& Bertelli, G. 2000, MNRAS, 318, 658

Binney, J., \& Merrifield, M. 1998, Galactic Astronomy (Princeton, NJ: Princeton University Press)

Binney, J., \& Tremaine, S. 1987, Galactic dynamics (Princeton, NJ: Princeton University Press)
Coulson, I. M., Walther, D. M., \& Dent, W. R. F. 1998, MNRAS, 296, 934

Decin, G., Dominik, C., Waters, L. B. F. M., \& Waelkens, C. 2003, ApJ, 598, 636

Dehnen, W., \& Binney, J. J. 1998, MNRAS, 298, 387

Dominik, C., \& Decin, G. 2003, ApJ, 598, 626

Emerson, J. P. 1988, in Formation and Evolution of Low Mass Stars, NATO ASIC Proc., 241, 193

ESA 1997, The Hipparcos and Tycho Catalogues, 1239

Habing, H. J., Dominik, C., Jourdain de Muizon, M., et al. 1999, Nature, 401, 456

Haisch, K. E., Lada, E. A., \& Lada, C. J. 2001, ApJ, 553, L153

Herbig, G. H., \& Simon, T. 2001, AJ, 121, 3138

Holland, W. S., Greaves, J. S., Zuckerman, B., et al. 1998, Nature, 392,788

Jahreiß, H., \& Wielen, R. 1983, in Nearby Stars and the Stellar Luminosity Function, IAU Coll., 76, 277

Jura, M. 1999, ApJ, 515, 706

Kalas, P., Graham, J. R., Beckwith, S. V. W., Jewitt, D. C., \& Lloyd, J. P. 2002, ApJ, 567, 999

Kenyon, S. J., \& Bromley, B. C. 2002, ApJ, 577, L35

Lachaume, R., Dominik, C., Lanz, T., \& Habing, H. J. 1999, A\&A, 348,897

Lada, E. A. 1999, in The Origin of Stars and Planetary Systems, NATO ASIC Proc., 540, 441

Lagrange, A.-M., Backman, D. E., \& Artymowicz, P. 2000, Protostars and Planets IV, 639

Lisse, C., Schultz, A., Fernandez, Y., et al. 2002, ApJ, 570, 779

Malfait, K., Bogaert, E., \& Waelkens, C. 1998, A\&A, 331, 211

Mundy, L. G., Looney, L. W., \& Welch, W. J. 2000, Protostars and Planets IV, 355

Oudmaijer, R. D., van der Veen, W. E. C. J., Waters, L. B. F. M., et al. 1992, A\&AS, 96, 625

Plets, H., Waelkens, C., Oudmaijer, R. D., \& Waters, L. B. F. M. 1997, A\&A, 323, 513

Pollack, J. B., Hubickyj, O., Bodenheimer, P., et al. 1996, Icarus, 124, 62

Silverstone, M. D. 2000, Ph.D. Thesis, UCLA

Song, I. 2000, Ph.D. Thesis, University of Georgia

Song, I. 2001, in Young Stars Near Earth: Progress and Prospects, ASP Conf. Ser., 244, 221

Spangler, C., Sargent, A. I., Silverstone, M. D., Becklin, E. E., \& Zuckerman, B. 2001, ApJ, 555, 932

Spitzer, L. J., \& Schwarzschild, M. 1951, ApJ, 114, 385

Spitzer, L. J., \& Schwarzschild, M. 1953, ApJ, 118, 106

Sylvester, R. J., \& Mannings, V. 2000, MNRAS, 313, 73

Thé, P. S., de Winter, D., \& Perez, M. R. 1994, A\&AS, 104, 315

van den Ancker, M. E., de Winter, D., \& Tjin A Djie, H. R. E. 1998, A\&A, 330, 145

Weidenschilling, S. J., \& Cuzzi, J. N. 1993, in Protostars and Planets III, 1031

Wielen, R. 1977, A\&A, 60, 263

Wilner, D. J., \& Lay, O. P. 2000, Protostars and Planets IV, 509

Zuckerman, B. 2001, ARA\&A, 39, 549

Zuckerman, B., \& Becklin, E. E. 1993, ApJ, 414, 793

Zuckerman, B., \& Song, I. 2004, ApJ, 603, 738 